\title{
Plasmon-Enhanced Energy Transfer in Photosensitive Nanocrystal Device
}

Shahab Akhavan, ${ }^{\dagger, \S, \#}$ Mehmet Zafer Akgul, ${ }^{\dagger, \|, \#}$ Pedro Ludwig Hernandez-Martinez, ${ }^{\dagger,+, \#}$

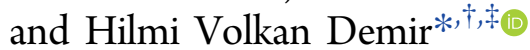

${ }^{\dagger}$ UNAM-Institute of Materials Science and Nanotechnology, Department of Electrical and Electronics Engineering and Department of Physics, Bilkent University, Ankara 06800, Turkey

${ }^{\ddagger}$ LUMINOUS! Center of Excellence, School of Electrical and Electronic Engineering and School of Physical and Mathematical Sciences, Nanyang Technological University, Singapore 639798, Singapore

Supporting Information
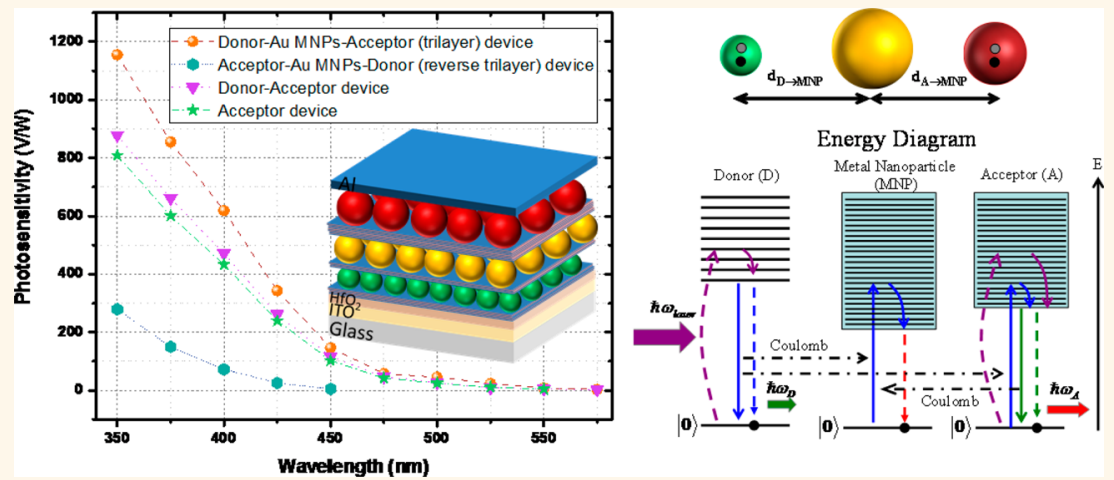

ABSTRACT: Förster resonance energy transfer (FRET) interacted with localized surface plasmon (LSP) gives us the ability to overcome inadequate transfer of energy between donor and acceptor nanocrystals (NCs). In this paper, we show LSP-enhanced FRET in colloidal photosensors of NCs in operation, resulting in substantially enhanced photosensitivity. The proposed photosensitive device is a layered self-assembled colloidal platform consisting of separated monolayers of the donor and the acceptor colloidal NCs with an intermediate metal nanoparticle (MNP) layer made of gold interspaced by polyelectrolyte layers. Using LBL assembly, we fabricated and comparatively studied seven types of such NC-monolayer devices (containing only donor, only acceptor, Au MNP-donor, Au MNP-acceptor, donor-acceptor bilayer, donor-Au MNP-acceptor trilayer, and acceptor-Au MNP-donor reverse trilayer). In these structures, we revealed the effect of LSPenhanced FRET and exciton interactions from the donor NCs layer to the acceptor NCs layer. Compared to a single acceptor NC device, we observed a significant extension in operating wavelength range and a substantial photosensitivity enhancement (2.91-fold) around the LSP resonance peak of Au MNPs in the LSP-enhanced FRET trilayer structure. Moreover, we present a theoretical model for the intercoupled donor-Au MNP-acceptor structure subject to the plasmonmediated nonradiative energy transfer. The obtained numerical results are in excellent agreement with the systematic experimental studies done in our work. The potential to modify the energy transfer through mastering the excitonplasmon interactions and its implication in devices make them attractive for applications in nanophotonic devices and sensors.

KEYWORDS: nanocrystals, semiconductor quantum dots, photosensors, localized plasmons, nonradiative energy transfer, excitons, LBL assembly

$\mathrm{F}$ örster resonance energy transfer (FRET), the transfer of the excitons from the donor particles to the acceptor particles by nonradiative dipole-dipole interactions, ${ }^{1,2}$ has been widely used for numerous applications including color tuning, ${ }^{3-5}$ white-light generation, ${ }^{6,7}$ nanosensors, ${ }^{8,9}$ and lightharvesting. ${ }^{10,11}$ Semiconductor nanocrystals (NCs), dubbed as colloidal quantum dots, are praised in FRET studies for their spectral tunability and precise control of peak emission resulting from the quantum confinement effect. Long-range interparticle dipole-dipole interaction of the donor (with a wider bandgap) emission to the absorption of the acceptor NCs (with a narrower bandgap) enables efficient energy transfer.

Received: December 14, 2016

Accepted: May 20, 2017

Published: May 20, 2017 
(a)

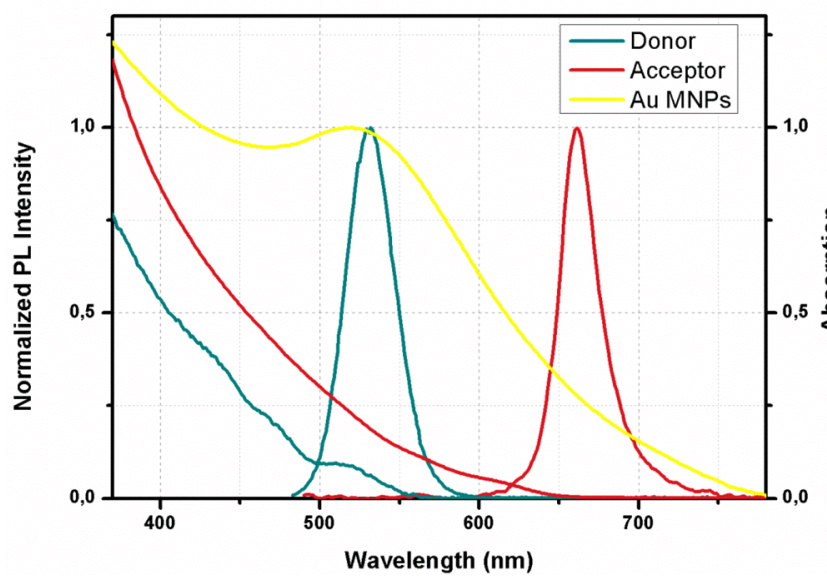

(b)

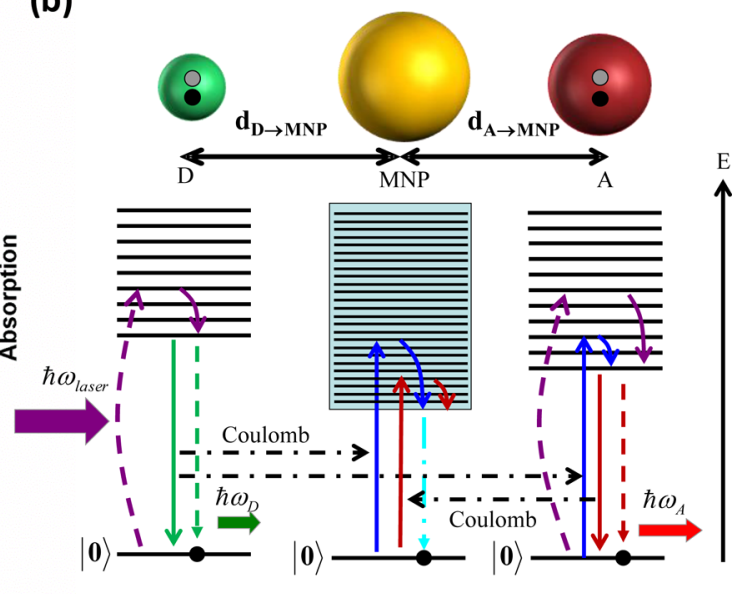

Figure 1. (a) In solution PL and absorption spectra of the donor NCs (celadon green line), the acceptor NCs (red line), and the optical absorption spectra for the Au MNPs (yellow line). (b) Schematic representation of a donor-Au MNPs-acceptor (trilayer) in which LSP supported by MNP-mediated energy transfer. The discrete energy levels for the NCs (left and right) and localized plasmon within the continuous band energy of the MNP (center) are shown in the energy band diagram. Light emission process (green and red line), absorption process (purple dash line), fast relaxation process (purple and blue short line), nonradiative relaxation process for the NCs (green, red dash line), nonradiative relaxation process for the MNP (cyan dash line), and energy transfer from the donor NCs to the acceptor NCs and the donor NCs to the MNPs pairs, due to the Coulomb interaction, are shown in black horizontal dash-dot lines. Exciton representation in the NCs (black and gray circles).

However, FRET efficiency suffers from a limited length scale of approximately $10 \mathrm{~nm}$ because of strong distance dependence of this process. Relatively large NCs diameter, which physically increases the donor-acceptor center to center separation, ${ }^{12,13}$ and inhomogeneous broadening of the NCs, which leads to intraensemble energy transfer, ${ }^{14,15}$ decrease the donor-acceptor FRET efficiency. Recently, it has been demonstrated that local electric field enhancement resulting from the localized surface plasmon (LSP) near a metal nanoparticle (MNP) can increase the strength of the donor-acceptor interaction, help to overcome the limit of the long center-to-center separation, and enhance the exciton transfer efficiency. ${ }^{16}$ In this regard, it is predicted that alteration of FRET mechanism with plasmonic structures offers great potential for FRET-based bioimaging and FRET-driven nanophotonic device applications. ${ }^{17,18}$

LSP-interacted FRET between donor-acceptor pairs has gained a strong interest and has been theoretically and experimentally explored. ${ }^{19-27}$ LSP-interacted FRET has been observed in core-shell structures, ${ }^{28,29}$ planar structures, ${ }^{30,31}$ and a single donor-acceptor pair coupled by single MNPs. ${ }^{32}$ Up to $70 \%$ enhancement in the Förster radius and 2 orders of magnitude increase in the FRET rate have been observed in LSP-enhanced FRET from a conjugated polymer to fluorescent multilayer core-shell NPs. ${ }^{33}$ However, a few works explore this mechansim in any kind of optoelectronic applications. ${ }^{34,35}$

More recently, LSP-enhanced FRET has been investigated in layered structures with separated donor NCs, acceptor NCs, and plasmonic nanostructures. ${ }^{17,30,36}$ Layered structure provides the opportunity to easily control the separations and ordering of the layers. A. L. Bradely et al. reported that positioning MNPs between the donor and the acceptor NCs and optimizing the separation distance result in large LSPenhanced FRET rate. ${ }^{30}$ Furthermore, better LSP-enhanced FRET interaction has been demonstrated from the photoluminescence $(\mathrm{PL})$ point of view by placing the plasmonic MNPs layer in the proximity of the donors layer while being sufficiently far away from the acceptors layer. ${ }^{18}$
In this article, we showed layered plasmonic and nonradiative energy-transfer interactions in a photovoltage controlled device by coupling MNPs to the donor-acceptor NCs dipolar interaction and enhancing excitons transfer from the donor to the acceptor NCs during the operation of the device. Here, we presented an NC-based photosensor that relies on photovoltage build-up readout for light-sensing applications. This device does not require any external bias, but instead operates based on photogenerated voltage build-up, unlike conventional charge collection devices. Therefore, enhancement or detraction in excitons generations due to the LSP-interacted FRET can be easily monitored in the proposed architecture. Moreover, we analyzed the changes in NCs emission under the LSP-interacted FRET condition and systematically studied the enhancement in energy-transfer efficiency within our structures. In addition, we proposed a theoretical model to elucidate underlying physics behind the experimental results. The numerical results showed the enhancement in LSPenhanced FRET efficiency, which was in excellent agreement with the systematic experimental measurements.

\section{RESULTS AND DISCUSSION}

The devices were assembled via subnanometer precision by layer-by-layer (LBL) technique (Supporting Information Figure S1). Similarly, all layered structures for PL and time-resolved fluorescence (TRF) measurements were prepared with the same recipe used for devices. The structures consisted of the donor NCs, the acceptor NCs, and the Au MNPs, each interspaced by a polyelectrolyte spacer layer. The thickness of the PDDA-PSS bilayer was approximately $1.2 \mathrm{~nm}$ as verified by the optical ellipsometry measurement. Details on the assembly procedures were explained in our previous works. ${ }^{37,38}$ In this work, highly luminescent and stable CdSe/CdZnS NCs with diameters of 4.80 and $6.20 \mathrm{~nm}$ were synthesized following a modified recipe of $\mathrm{W}$. K. Bae et $a l^{39}$ as the donor and the acceptor NCs, respectively. As-synthesized NCs were ligand exchanged using negatively charged mercaptopropionic acid, 

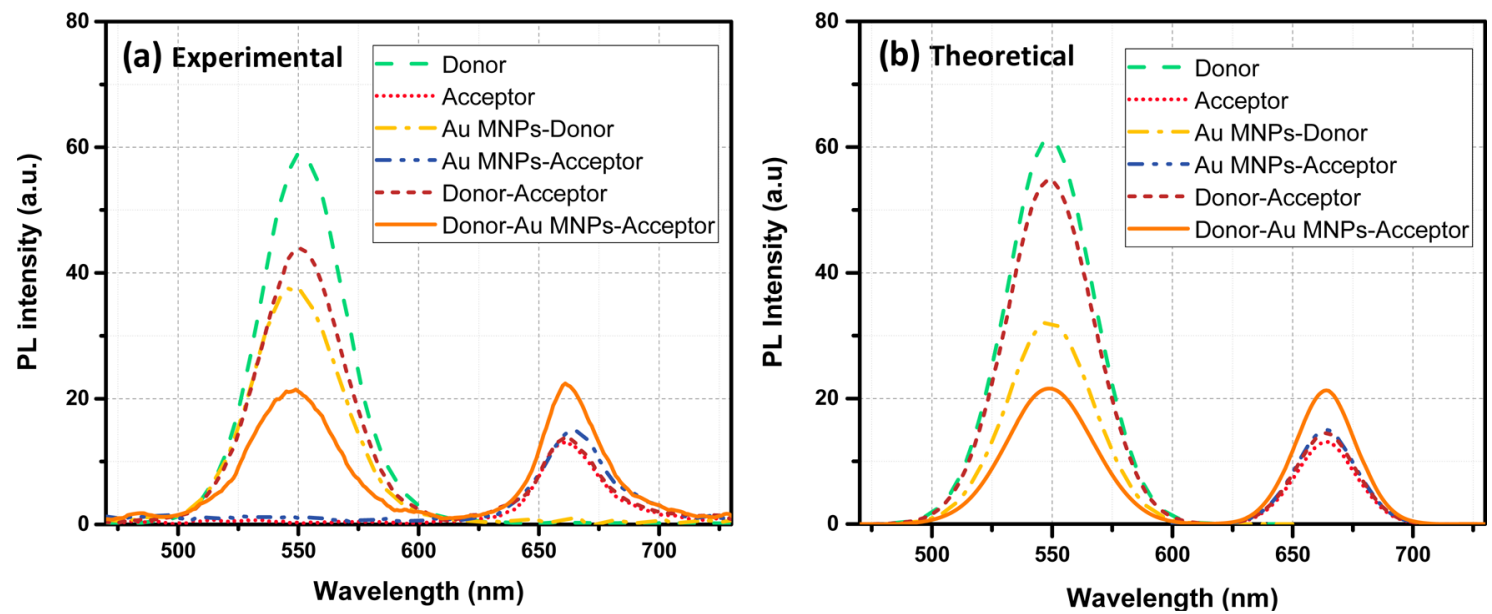

Figure 2. (a) Experimental and (b) calculated PL spectra of films based on the donor (dash line), the acceptor (short dot line), the donoracceptor bilayer (short dash line), the Au MNPs-donor (dash dot line), the Au MNPs-acceptor (dash dot dot line), and the donor-Au MNP-acceptor layered structure (solid line). The numerical results are in good agreement with the experimental measurements.

which yielded stable NCs in aqueous solution (Supporting Information Figure S2). Negatively charged thioglycolic acidcapped $\mathrm{Au}$ MNPs with an average diameter of around $5 \mathrm{~nm}$ were prepared following the modified synthesis recipe of M. N. Martin et al. ${ }^{40}$ (Supporting Information Figure S2). The synthesized Au MNPs showed high stability in solution, which allowed the preparation of a well-defined monolayer structure during the LBL process. Furthermore, MNPs of this size can be well processed into closely packed monolayers with welldefined vertical thickness, which would not be possible with larger gold MNPs. Indeed, larger nanoparticles suffer from low concentration in solution, resulting in low-density and rough monolayer topography, which is undesirable for LSP-interacted FRET studies.

The donor and the acceptor NCs have an emission peak at 530 and $661 \mathrm{~nm}$ in solution, respectively. Absorption and emission spectra of the donor, the acceptor, and the Au MNPs are provided in Figure 1a (see amplitude-averaged lifetime of NCs in Supporting Information Figure S3). It can clearly be seen that the donor and the acceptor have spectrally separated PL emission spectra and the donor emission overlaps well with the acceptor absorption, enabling efficient resonant energy transfer between the donor and the acceptor NCs. Moreover, the LSP resonance of the Au MNP is visible as a peak at 523 $\mathrm{nm}$ in the absorption spectra, which overlaps with the donor emission peak, see Figure 1a.

To verify the existence of LSP-enhanced FRET, we conducted PL and TRF spectroscopy of the trilayer structure as well as the reference donor, acceptor, donor-acceptor bilayer, and $\mathrm{Au}$ MNPs-NCs bilayers. Here, the trilayer structure was based on donor-Au MNPs-acceptor (Figure $1 \mathrm{~b})$, with surface-to-surface interspace of $5.2 \mathrm{~nm}$ in donor-Au MNPs and surface-to-surface interspace of approximately 10 $\mathrm{nm}$ in Au MNPs-acceptor. This exhibited the largest reduction in the donor PL lifetime along with the enhancement in acceptor PL emission and the largest LSP enhancement of FRET between the donor and the acceptor NC layer, in accordance with refs 18 and 30 .

PL spectra of all prepared NC films are demonstrated in Figure 2a. First, we prepared a monolayer of the donor and the acceptor structures. In these samples, one monolayer $(1 \mathrm{ML})$ of $\mathrm{NC}$ is deposited on top of four bilayers of polyelectrolyte polymers. In order to get a film of NC monolayer with enough surface coverage and uniformity, we noticed that four bilayers of PDDA-PSS were necessary. The control groups for plasmonics were set as follows: The donor NCs were plasmon-interacted to $1 \mathrm{ML}$ of Au MNPs interspaced by 3 MLs of PDDA/PSS pair followed by a final single layer of PDDA. Similarly, for plasmon coupling to an acceptor, $1 \mathrm{ML}$ of $\mathrm{Au}$ MNPs was interspaced from $1 \mathrm{ML}$ of acceptor NC by 7 MLs of PDDA/PSS pair, followed by a final single layer of PDDA. As can be seen in Figure 2a, because of the close range between donor NCs and Au MNPs, the donor emission was quenched by $31 \%$, ascribing to an increase in nonradiative processes. In contrast, in the case of sufficient distance between Au MNPs and acceptor NCs, we observed emission enhancement of the acceptor NCs by $35 \%$ because of the local electric field enhancement generated by the MNPs. ${ }^{41,42}$

For the only FRET control group, $1 \mathrm{ML}$ of the donor NCs was interspaced from single acceptor NCs layer by 17 MLs of PDDA/PSS pair, followed by $1 \mathrm{ML}$ of PDDA. In this case, the donor emission was reduced by $25 \%$, while the emission of the acceptor was slightly increased by $6 \%$. Next, we prepared a trilayer structure where a single donor NCs layer was separated from single layer of Au MNPs layer by 3 MLs of PDDA/PSS pair plus $1 \mathrm{ML}$ of PDDA. Likewise, $1 \mathrm{ML}$ of the acceptor NCs was interspaced by 7 MLs of PDDA/PSS pair + 1 ML PDDA from the Au MNPs. Figure 2a shows that the acceptor emission in the trilayer structure undergoes a significant enhancement of $68 \%$, whereas the PL intensity in the donor NCs is reduced by 64\%. In all cases, the PL enhancement/quenching is calculated from the integrated deconvolved donor/acceptor spectra.

To further understand the plasmon-coupled FRET, we develop a theoretical model to describe the experimental results. We separate our problem into three cases: (1) FRET in donor-acceptor NCs; (2) plasmon-enhanced (donor/acceptor) NCs; and (3) plasmon-interacted FRET donor-Au MNPs-acceptor. Here, the main resulting interactions are exciton-exciton and exciton-plasmon interactions (see Supporting Information Figure S4). First, we estimate FRET rate in the donor-acceptor NCs considering the case of a single donor NC to a two-dimensional (2D) layer of acceptor NCs: 
Table 1. Experimental and Theoretical Analysis Results ${ }^{a}$

\begin{tabular}{|c|c|c|c|c|c|c|c|}
\hline \multicolumn{8}{|c|}{ time-resolved analysis (experimental) } \\
\hline$\tau_{\mathrm{D}}$ & $\tau_{\mathrm{d}(\mathrm{D}-\mathrm{A})}$ & $\mathrm{Au}-\mathrm{D}$ & $\tau_{\mathrm{d}(\mathrm{D}-\mathrm{Au}-\mathrm{A})}$ & $\gamma_{\text {FRET }}$ & $\gamma_{\text {LSP-FRET }}$ & $\eta_{\text {FRET }}$ & $\eta_{\text {LSP-FRET }}$ \\
\hline 10.08 & 7.52 & .44 & 3.13 & 0.03 & 0.22 & 0.25 & 0.69 \\
\hline$\tau_{\mathrm{A}}$ & $\tau_{\mathrm{a}(\mathrm{D}-\mathrm{A})}$ & $\mathrm{Au}-\mathrm{A}$ & \multirow{2}{*}{$\begin{array}{c}\tau_{\mathrm{a}(\mathrm{D}-\mathrm{Au}-\mathrm{A})} \\
7.01\end{array}$} & $\gamma_{\text {FRET }}$ & $\gamma_{\text {LSP-FRET }}$ & $\eta_{\text {FRET }}$ & $\eta_{\text {LSP-FRET }}$ \\
\hline 5.43 & 6.27 & 1.72 & & & & & \\
\hline \multicolumn{8}{|c|}{ steady-state analysis (experimental) } \\
\hline$I_{\mathrm{D}}$ & $I_{\mathrm{d}(\mathrm{D}-\mathrm{A})}$ & $I_{\mathrm{Au}-\mathrm{D}}$ & $I_{\mathrm{d}(\mathrm{D}-\mathrm{Au}-\mathrm{A})}$ & $\varsigma \mathrm{d}(\mathrm{D}-\mathrm{A}) / \mathrm{D}$ & \multicolumn{2}{|c|}{$\varsigma_{\mathrm{Au}-\mathrm{D} / \mathrm{D}}$} & $\zeta \mathrm{d}(\mathrm{D}-\mathrm{Au}-\mathrm{A}) / \mathrm{D}$ \\
\hline 2626 & 1960 & 1823 & 941 & 0.75 & \multicolumn{2}{|c|}{0.69} & 0.36 \\
\hline$I_{\mathrm{A}}$ & $I_{\mathrm{a}(\mathrm{D}-\mathrm{A})}$ & $I_{\mathrm{Au}-\mathrm{A}}$ & $I_{\mathrm{a}(\mathrm{D}-\mathrm{Au}-\mathrm{A})}$ & $\varsigma_{\mathrm{a}(\mathrm{D}-\mathrm{A}) / \mathrm{A}}$ & \multicolumn{2}{|c|}{$\varsigma_{\mathrm{Au}-\mathrm{A} / \mathrm{A}}$} & $\varsigma_{\mathrm{a}(\mathrm{D}-\mathrm{Au}-\mathrm{A}) / \mathrm{A}}$ \\
\hline 478 & 507 & 645 & 803 & 1.06 & \multicolumn{2}{|c|}{1.35} & 1.68 \\
\hline \multicolumn{8}{|c|}{ steady-state analysis (theoretical) } \\
\hline$I_{\mathrm{D}}$ & $I_{\mathrm{d}(\mathrm{D}-\mathrm{A})}$ & $I_{\mathrm{Au}-\mathrm{D}}$ & $I_{\mathrm{d}(\mathrm{D}-\mathrm{Au}-\mathrm{A})}$ & $\varsigma_{\mathrm{d}(\mathrm{D}-\mathrm{A}) / \mathrm{D}}$ & \multirow{2}{*}{\multicolumn{2}{|c|}{$\begin{array}{c}\varsigma_{\mathrm{Au}-\mathrm{D} / \mathrm{D}} \\
0.53\end{array}$}} & $\zeta_{\mathrm{d}(\mathrm{D}-\mathrm{Au}-\mathrm{A}) / \mathrm{D}}$ \\
\hline 2784 & 2487 & 1462 & 981 & 0.89 & & & 0.35 \\
\hline$I_{\mathrm{A}}$ & $I_{\mathrm{a}(\mathrm{D}-\mathrm{A})}$ & $I_{\mathrm{Au}-\mathrm{A}}$ & $I_{\mathrm{a}(\mathrm{D}-\mathrm{Au}-\mathrm{A})}$ & $\varsigma_{\mathrm{a}(\mathrm{D}-\mathrm{A}) / \mathrm{A}}$ & \multicolumn{2}{|c|}{$\varsigma_{\mathrm{Au}-\mathrm{A} / \mathrm{A}}$} & $\varsigma_{\mathrm{a}(\mathrm{D}-\mathrm{Au}-\mathrm{A}) / \mathrm{A}}$ \\
\hline 418 & 463 & 479 & 680 & 1.10 & \multicolumn{2}{|c|}{1.15} & 1.63 \\
\hline$\lambda_{\mathrm{D}}$ & amplitude-averaged lifetime (ns) & $t_{1}(\mathrm{~ns})$ & $t_{2}(\mathrm{~ns})$ & $t_{3}(\mathrm{~ns})$ & $A_{1}$ & $A_{2}$ & $A_{3}$ \\
\hline $\mathrm{D}$ & 10.08 & $40.513 \pm 0.703$ & $13.888 \pm 0.126$ & $5.260 \pm 0.147$ & $664.9 \pm 17.3$ & $5165.2 \pm 58.4$ & $4819.0 \pm 128.0$ \\
\hline $\mathrm{D}-\mathrm{A}$ & 7.52 & $33.954 \pm 0.526$ & $13.328 \pm 0.139$ & $4.595 \pm 0.140$ & $773.3 \pm 18.7$ & $4166.8 \pm 54.2$ & $4414 \pm 130$ \\
\hline $\mathrm{Au}-\mathrm{D}$ & 5.44 & $29.384 \pm 0.553$ & $10.415 \pm 0.122$ & $3.5299 \pm 0.095$ & $570.1 \pm 17.0$ & $3872.7 \pm 58.5$ & $5528 \pm 147$ \\
\hline $\mathrm{D}-\mathrm{Au}-\mathrm{A}$ & 3.13 & $28.435 \pm 0.701$ & $8.291 \pm 0.116$ & $2.6191 \pm 0.082$ & $349.1 \pm 13.3$ & $3189.3 \pm 58.0$ & $4999 \pm 160$ \\
\hline$\lambda_{\mathrm{A}}$ & amplitude-averaged lifetime (ns) & $t_{1}(\mathrm{~ns})$ & $t_{2}(\mathrm{~ns})$ & $t_{3}(\mathrm{~ns})$ & $A_{1}$ & $A_{2}$ & $A_{3}$ \\
\hline A & 5.43 & $147.39 \pm 8.25$ & $13.502 \pm 0.214$ & $3.8346 \pm 0.076$ & $63.81 \pm 2.76$ & $1437.2 \pm 31.6$ & $4880 \pm 105$ \\
\hline $\mathrm{D}-\mathrm{A}$ & 6.27 & $51.79 \pm 1.56$ & $15.295 \pm 0.221$ & $4.043 \pm 0.131$ & $220.0 \pm 9.37$ & $1886.4 \pm 34.0$ & $3283 \pm 106$ \\
\hline $\mathrm{Au}-\mathrm{A}$ & 4.72 & $144.9 \pm 10.3$ & $13.822 \pm 0.290$ & $3.6012 \pm 0.069$ & $67.76 \pm 2.73$ & $963.7 \pm 27.7$ & $4915 \pm 105$ \\
\hline $\mathrm{D}-\mathrm{Au}-\mathrm{A}$ & 7.01 & $111.06 \pm 3.87$ & $14.920 \pm 0.196$ & $4.1080 \pm 0.074$ & $115.69 \pm 3.81$ & $1913.6 \pm 34.1$ & $5919 \pm 113$ \\
\hline
\end{tabular}

${ }^{a} \mathrm{D}, \mathrm{A}, \mathrm{D}-\mathrm{A}, \mathrm{Au}-\mathrm{D}, \mathrm{Au}-\mathrm{A}$, and $\mathrm{D}-\mathrm{Au}-\mathrm{A}$ stands for single donor NCs layer, single acceptor NCs layer, donor-acceptor bilayer, Au MNPs-donor NCs bilayer, Au MNPs-acceptor NCs bilayer, and donor-Au MNPs-acceptor trilayer. $\tau_{\mathrm{D}}$ : Amplitude-averaged lifetime of single donor NCs layer at donor emission wavelength. $\tau_{\mathrm{d}(\mathrm{D}-\mathrm{A})}$ : amplitude-averaged lifetime of donor-acceptor bilayer at donor emission wavelength. $\tau_{\mathrm{Au}-\mathrm{D}}$ : Amplitudeaveraged lifetime of Au MNPs-donor NCs bilayer at donor emission wavelength. $\tau_{\mathrm{d}(\mathrm{D}-\mathrm{Au}-\mathrm{A})}$ : Amplitude-averaged lifetime of donor-Au MNPsacceptor trilayer at donor emission wavelength. $\tau_{\mathrm{A}}$ : Amplitude-averaged lifetime of single acceptor NCs layer at acceptor emission wavelength. $\tau_{\mathrm{a}(\mathrm{D}-\mathrm{A})}:$ Amplitude-averaged lifetime of donor-acceptor bilayer at acceptor emission wavelength. $\tau_{\mathrm{Au}-\mathrm{A}}$ : Amplitude-averaged lifetime of Au MNPsacceptor NCs bilayer at acceptor emission wavelength. $\tau_{\mathrm{a}(\mathrm{D}-\mathrm{Au}-\mathrm{A})}$ : Amplitude- averaged lifetime of donor-Au MNPs-acceptor trilayer at acceptor emission wavelength. $\gamma_{\mathrm{FRET}}$ : Energy-transfer rate because of FRET. $\gamma_{\mathrm{LSP} \text {-FRET }}$ : Energy-transfer rate owing to FRET and localized surface plasmonic interactions. $\eta_{\mathrm{FRET}}$ : Energy-transfer efficiency because of FRET. $\eta_{\mathrm{LSP}-\mathrm{FRET}}$ : Energy-transfer efficiency owing to FRET and localized surface plasmonic interactions. I: Integrated emission intensity. ऽ: Change in steady-state PL.

$$
\gamma_{\mathrm{FRET}}=\gamma_{\mathrm{D}}\left(\frac{\pi}{2}\right) \sigma_{\mathrm{A}} \frac{R_{0}^{6}}{d^{4}}
$$

where $R_{0}$ is the Förster radius; ${ }^{43} \gamma_{\mathrm{D}}=\gamma_{\mathrm{D}, \mathrm{r}}+\gamma_{\mathrm{D}, \mathrm{nr}}=1 / \tau_{\mathrm{D}}$ is the donor exciton recombination rate; $\sigma_{\mathrm{A}}$ is the number of acceptor per unit area; and $d$ is the donor-acceptor center-to-center separation distance. The respective emission enhancement factors are

$$
\begin{aligned}
\kappa_{\mathrm{D}, \mathrm{FRET}} & =\frac{\gamma_{D}}{\gamma_{\mathrm{D}}+\gamma_{\mathrm{FRET}}} \\
\kappa_{\mathrm{A}, \mathrm{FRET}} & =1+\frac{\gamma_{\mathrm{A}}}{\gamma_{\mathrm{A}}+\gamma_{\mathrm{FRET}}}
\end{aligned}
$$

Next, we consider the case of NCs interacting with MNPs. The energy-transfer rate from a single NC to a $2 \mathrm{D} M N P$ array is ${ }^{44}$

$$
\gamma_{\mathrm{nr}, \text { metal }, \alpha}=\frac{2}{\hbar} b_{\alpha}\left(\frac{e d_{\mathrm{exc}}}{\varepsilon_{\mathrm{eff}}}\right)^{2} \frac{\pi}{2} \sigma_{\mathrm{MNP}}\left(\frac{R_{\mathrm{MNP}}^{3}}{d^{4}}\right)\left|\frac{3 \varepsilon_{0}}{2 \varepsilon_{0}+\varepsilon_{\mathrm{MNP}}(\omega)}\right|^{2} \operatorname{Im}\left|\varepsilon_{\mathrm{MNP}}(\omega)\right|
$$

where $b_{\alpha}=\frac{1}{3}, \frac{1}{3}, \frac{4}{3}$ for $\alpha=x, y, z$, respectively; $\varepsilon_{0}$ is the medium dielectric constant; $\varepsilon_{\mathrm{MNP}}(\omega)$ is the Au dielectric function; $\sigma_{\mathrm{MNP}}$ is the number of Au MNP per unit area; $R_{\mathrm{MNP}}$ is the radius of $\mathrm{Au} \mathrm{MNP;} d_{\mathrm{exc}}$ is the donor NC exciton dipole moment; $d$ is the NCs-Au MNPs center-to-center separation distance; and $\varepsilon_{\text {eff }}=\left(2 \varepsilon_{0}+\varepsilon_{\mathrm{NC}}\right) / 3$ is the effective dielectric constant. The total nonradiative-transfer rate from the NC to the MNP is defined as $\gamma_{\text {nr,metal }}=\left(\gamma_{x}+\gamma_{y}+\gamma_{z}\right) / 3$. We assume that $\gamma_{\mathrm{NC}, \mathrm{nr}}=\gamma_{0, \mathrm{NC}, \mathrm{nr}}$ does not change in the presence of MNPs, while the radiative rate and absorption intensity are modified by the MNPs as ${ }^{45}$

$$
\begin{aligned}
& \gamma_{\mathrm{NC}, \mathrm{r}}=A\left(\omega_{\mathrm{LSP}}\right) \gamma_{0, \mathrm{NC}, \mathrm{r}} \\
& I_{\mathrm{abs}}=A\left(\omega_{\mathrm{LSP}}\right) I_{0, \mathrm{abs}}
\end{aligned}
$$

where $\gamma_{0, \mathrm{NC}, \mathrm{r}}, \gamma_{0, \mathrm{NC}, \mathrm{nr}}$, and $I_{0, \mathrm{abs}}$ are radiative, nonradiative, and exciton generation rate in the absence of MNPs, respectively; $\omega_{\text {LSP }}$ is the localized surface plasmon frequency of the $\mathrm{Au}$ MNP; and $A(\omega)$ is the electric field enhancement factor termed as

$$
A(\omega)=\frac{\int_{\mathrm{NC}}\left|E_{\mathrm{in}, \mathrm{NC}}\right|^{2} d V}{\int_{\mathrm{NC}}\left|E_{0}\right|^{2} d V}
$$

where $E_{\mathrm{in}, \mathrm{NC}}, E_{0}$ is the electric field inside the NCs in the occurrence of MNPs and in the absence of MNPs, respectively. The emission enhancement factor $\left(\kappa_{\mathrm{LSP}}(\omega)\right)$ for the NC in the occurrence of MNPs is 

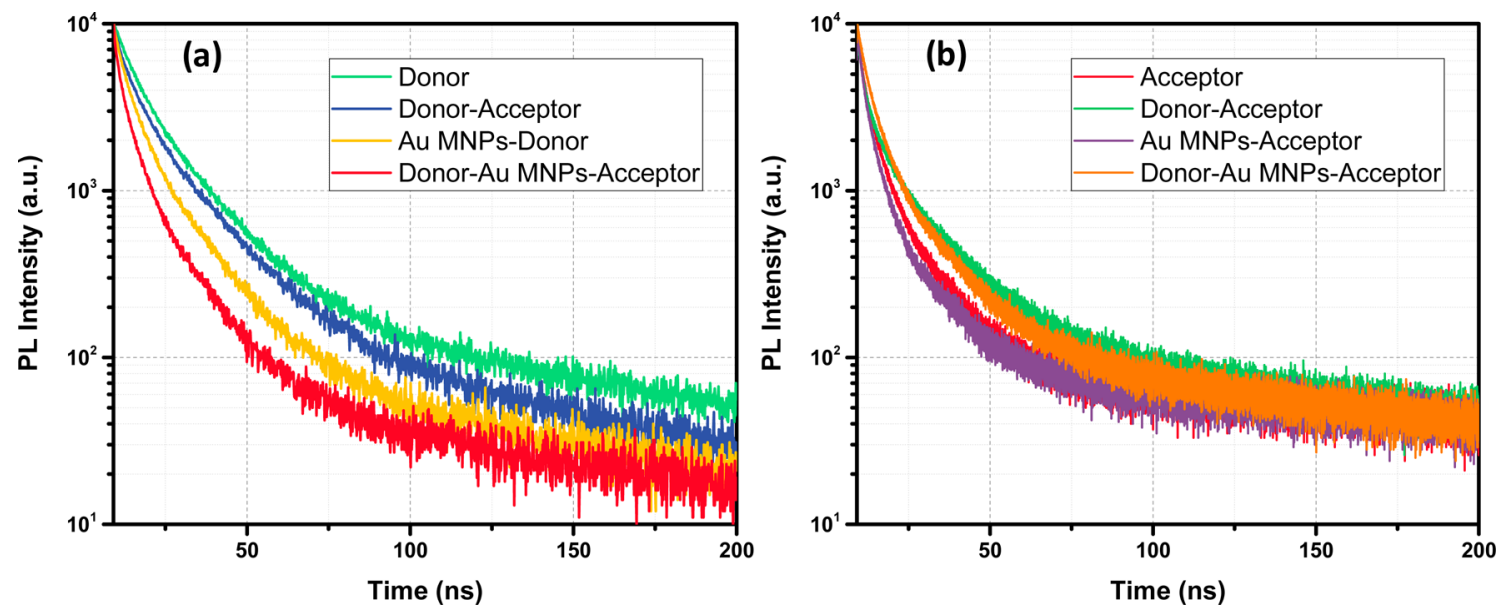

Figure 3. PL decay curves for (a) the donor, donor-acceptor bilayer, Au MNPs-donor, and donor-Au MNPs-acceptor at $550 \mathrm{~nm}$ and (b) the acceptor, donor-acceptor bilayer, Au MNPs-acceptor, and donor-Au MNPs-acceptor at $664 \mathrm{~nm}$. The figure shows a strong modification in the TRF caused by plasmon and/or FRET interaction.

$$
\kappa_{\mathrm{LSP}}\left(\omega_{\text {laser }}\right)=\frac{A\left(\omega_{\mathrm{LSP}}\right) \gamma_{\mathrm{NC}}}{\left[1+\left(A\left(\omega_{\mathrm{LSP}}\right)-1\right) Y_{\mathrm{NC}}\right] \gamma_{\mathrm{NC}}+\gamma_{\mathrm{nr}, \mathrm{Au}}}
$$

Finally, we calculate plasmon-interacted FRET for the case of donor-acceptor NCs in the presence of MNPs and estimate the emission intensity for the donor and the acceptor NCs. The energy-transfer rate between the donor (acceptor) - MNP is estimated as mentioned above. The enhancement in FRET efficiency is calculated for a single donor NC to a $2 \mathrm{D}$ plane of acceptor NCs as follows:

$$
\gamma_{\text {LSP-FRET }}=\gamma_{\mathrm{D}}\left(\frac{\pi}{2}\right) \sigma_{\mathrm{A}} \frac{R_{0, \mathrm{LSP}}{ }^{6}}{d^{4}}
$$

Here, we assume that the Förster radius is enhanced by

$$
R_{0, \mathrm{LSP}}=A_{\mathrm{D}}\left(\omega_{\mathrm{LSP}}\right) R_{0}
$$

This assumption is supported by refs 18 and 30, where it was experimentally shown that the Förster radius is enhanced by approximately a factor of $2\left(R_{0, \mathrm{LPS}} \sim 2 R_{0}\right)$ when the LSP of the MNPs are coupled to the donor NC. Thus, the donor (acceptor) emission enhancement factor is given by

$$
\kappa_{\mathrm{D}, \mathrm{LSP}-\mathrm{FRET}}=\frac{A_{\mathrm{D}}\left(\omega_{\mathrm{LSP}}\right) \gamma_{\mathrm{D}}}{\left[1+\left(A_{\mathrm{D}}\left(\omega_{\mathrm{LSP}}\right)-1\right) Y_{\mathrm{D}}\right] \gamma_{\mathrm{D}}+\gamma_{\mathrm{D}, \mathrm{nr}, \mathrm{Au}}+\gamma_{\mathrm{LSP}-\mathrm{FRET}}}
$$

$$
\begin{aligned}
& \kappa_{\mathrm{A}, \mathrm{LSP}-\mathrm{FRET}}=\frac{A_{\mathrm{A}}\left(\omega_{\mathrm{LSP}}\right) \gamma_{\mathrm{A}}}{\left[1+\left(A_{\mathrm{A}}\left(\omega_{\mathrm{LSP}}\right)-1\right) Y_{\mathrm{A}}\right] \gamma_{\mathrm{A}}+\gamma_{\mathrm{A}, \mathrm{nr}, \mathrm{Au}}} \\
& \times\left(1+\frac{\gamma_{\mathrm{LSP}-\mathrm{FRET}}}{\left[1+\left(A_{\mathrm{D}}\left(\omega_{\mathrm{LSP}}\right)-1\right) Y_{\mathrm{D}}\right] \gamma_{\mathrm{D}}+\gamma_{\mathrm{D}, \mathrm{nr}, \mathrm{Au}}+\gamma_{\mathrm{LSP}-\mathrm{FRET}}}\right. \\
& \left.\quad \times \frac{A_{\mathrm{D}}\left(\omega_{\mathrm{LSP}}\right) I_{0, \mathrm{D}, \mathrm{emiss}}}{A_{\mathrm{A}}\left(\omega_{\mathrm{LSP}}\right) I_{0, \mathrm{~A}, \mathrm{emiss}}}\right)
\end{aligned}
$$

The theoretical results are in good agreement with the experimental findings, however, there are slight differences between them as can be seen in Figure 2 and Table 1. These differences between experimental and theoretical calculations in the donor side of Au MNPs-donor and donor-acceptor NC bilayers can be referred to the differences between the assumptions made in theoretical and the experimental conditions. For instance, we consider that there is no energy transfer between the same species, i.e., donor-donor or acceptor-acceptor energy transfer. In addition, there is a possibility of partial detachment of the donor NCs during LBL assembly. The donor and acceptor NCs layer are interspaced with $17 \mathrm{MLs}$ of polyelectrolyte layers, which requires several consecutive dippings and rinsings that may result in fractional detachment of donor NC layers.

Further proof of LSP-enhanced FRET is found in the comparative study of the emission kinetics of structures using TRF. Figure 3a shows a strong modification in the TRF caused by plasmon and/or FRET interaction. For instance, the amplitude-averaged PL lifetime of the donor NCs layer decreased from 10.08 to $7.52 \mathrm{~ns}$ in the donor-acceptor bilayer structure, compared to the reference only donor structure. On the other hand, in the existence of Au MNPs, the donor lifetime decreased to 5.44 ns due to the plasmon resonance energy transfer. Finally, in the trilayer structure, the donor lifetime was further decreased to $3.13 \mathrm{~ns}$, indicating strong energy transfer. For the LSP-enhanced FRET system described above, the calculated energy-transfer rate was $0.22 \mathrm{~ns}^{-1}$, which is much faster than the FRET rate of $0.03 \mathrm{~ns}^{-1}$ in the absence of plasmon coupling. The plasmon coupled transfer rate for the trilayer structure was calculated by $\gamma_{\text {LSP-FRET }}=1 / \tau_{\mathrm{D}-\mathrm{Au}-\mathrm{A}}-1$ / $\tau_{\mathrm{D}}$. This indicated that the energy was transferred from the donors to the acceptors with an efficiency of $\eta=1-\tau_{\mathrm{D}-\mathrm{Au}-\mathrm{A}} /$ $\tau_{\mathrm{D}}=69 \%$. It is worth mentioning that the donor PL quenching of $64 \%$ is in excellent agreement with the LSP-enhanced FRET efficiency calculated from the donor lifetime.

At the acceptor side (see Figure $3 b$ ), the acceptor NCs amplitude-averaged lifetime was $5.43 \mathrm{~ns}$, whereas in the presence of $\mathrm{Au}$ MNPs, which were placed at a sufficiently long interval (7 PDDA/PSS pairs) from the acceptor NCs, the lifetime decreased to $4.72 \mathrm{~ns}$. Here, the increase in the local density of states near Au MNPs provide additional channels which increased the overall de-excitation decay rate. On the other hand, in the presence of the donor without Au MNPs, the acceptor NCs lifetime changed from 5.43 to $6.27 \mathrm{~ns}$ due to donor-acceptor Förster-type energy transfer. Finally, in the presence of LSP-enhanced FRET, we observed a further increase in the acceptor NCs lifetime to $7.01 \mathrm{~ns}$. This increment indicates a stronger energy transfer between all species.

Next, we investigate the influence of LSP-interacted FRET on the active operation of our colloidal photosensors, which we 
(a)
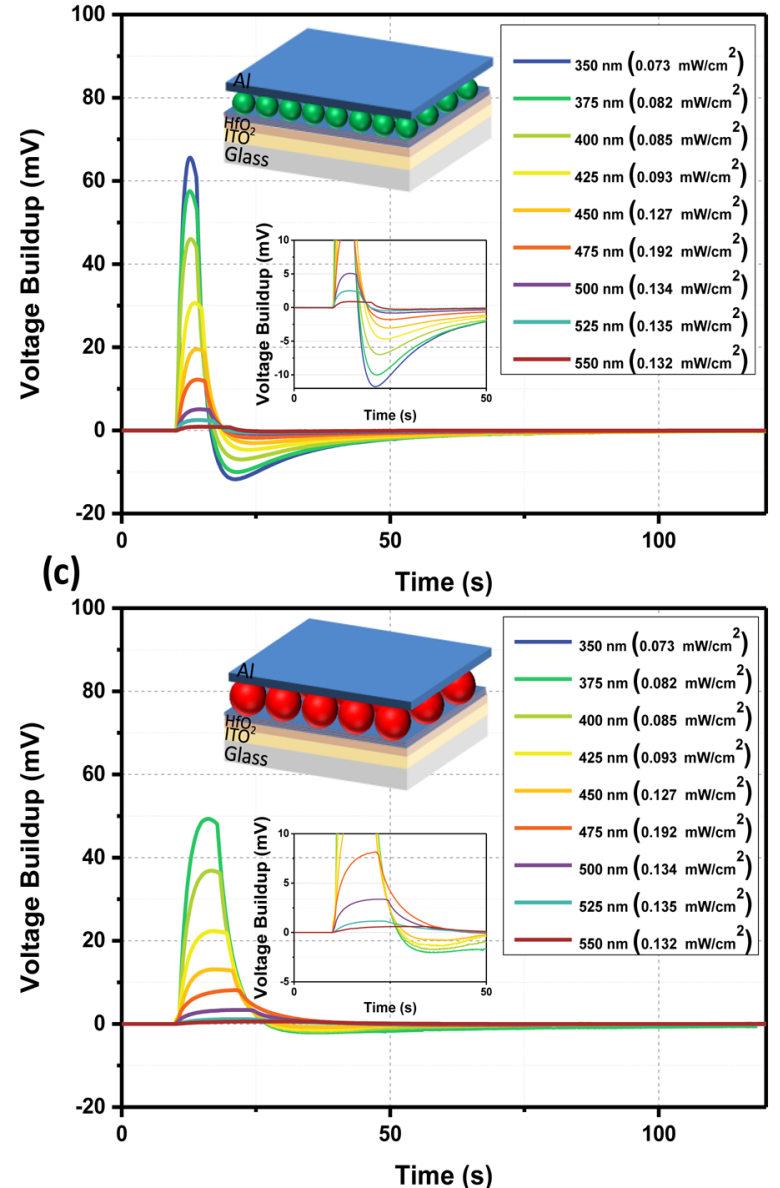

(b)
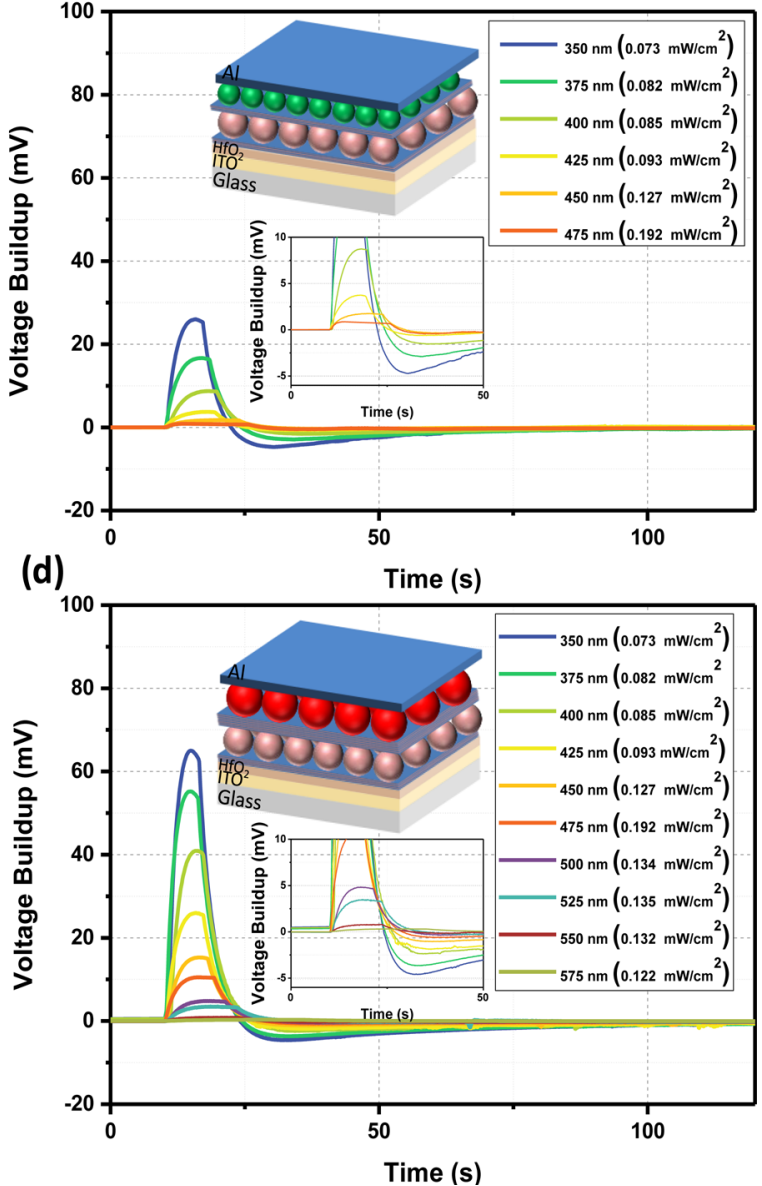

Figure 4. Photovoltage build-up vs time of PNMs at various excitation wavelengths and optical intensities for (a) single donor NC layer, (b) Au MNPs-donor, (c) single acceptor NC layer, and (d) Au MNPs-acceptor. All photovoltage build-up results in already reached steady-state conditions (peak point), and then incident light was turned off. Green, red, and light-pink spheres represent the donor, the acceptor, and the Au MNPs, respectively. Because of the quenching, there is a reduction in the voltage buildup of the Au MNP-donor device compared to the single donor NC layer device. However, in comparison to the only acceptor and Au MNPs-acceptor devices, the enhancement in the voltage build-up can be clearly seen.

coin photosensitive nanocrystal monolayers (PNMs) here. These PNMs can operate using only a single monolayer of NCs where across which no external bias is applied. Such an architecture is suitable for the utilization of layered structures where there is an increasing donor-acceptor NCs dipolar interaction via LSP and/or FRET. With light illumination on the NCs, most of the photogenerated excitons created inside the NCs are disassociated at the NC-Al interface. While electrons favor staying in the NCs, holes are transferred into the $\mathrm{Al}$ side because of the energy band alignment at the interface. These are essentially nontrapped and accumulated charges because of the capacitive nature of the PNM. Nonetheless, some of the photogenerated electrons can also be trapped and remained in the NCs. ${ }^{46,47}$

When the light impinges on the device, we first observe a net potential build-up. After reaching a peak point, this voltage build-up decreases as a function of time until it reaches a steady state in the presence of lighting. After that, when the incident light is turned off, a negative potential (voltage build-up) appears, and over time it returns to the initial level (see Supporting Information Figure S5). To elucidate the device's working dynamics, it is important to note that the nontrapped charges (accumulated charges) continuously discharge because of the shunt resistor (100 M $\Omega$ connected externally to the device) and the RC decay of the whole circuitry (the device + shunt resistor). Moreover, there are trapped charges in the NCs, which cause a negative potential in the absence of light. ${ }^{48,49}$ When the voltage build-up reaches a peak point, these opposing effects become equal and neutralize each other. By switching off the light at the peak point (see Figures 4 and 5), the photovoltage build-up appears to decay and goes negative. This negative value is due to the trapped charges. As a consequence, the negative voltage build-up gets to its lowest value and then comes back to zero (starting level). The time needs for the negative build-up to return zero is directly linked to the time required to deplete these trapped charges. In consequence, PNM can be counted as an open-circuit device that relies on photogenerated voltage build-up. As a figure of merit for the photosensitivity of the PNM, we calculated it according to the ratio of $S=V / P$, where $V$ is the photovoltage build-up (in $\mathrm{V}$ ) and $P$ is the incident optical power (in $\mathrm{W}$ ) impinging on the active area of the device.

To this end, we demonstrate that using the LSP-enhanced FRET, photovoltage build-up and consequently photosensitivity in PNM can be strongly enhanced, and largely broadband photosensitivity is achievable. Photovoltage build-up as a 
(a)

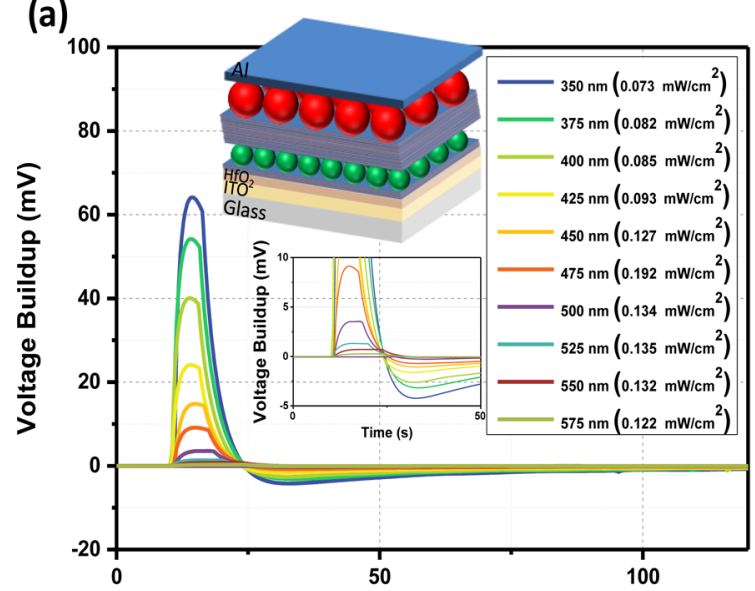

(c)

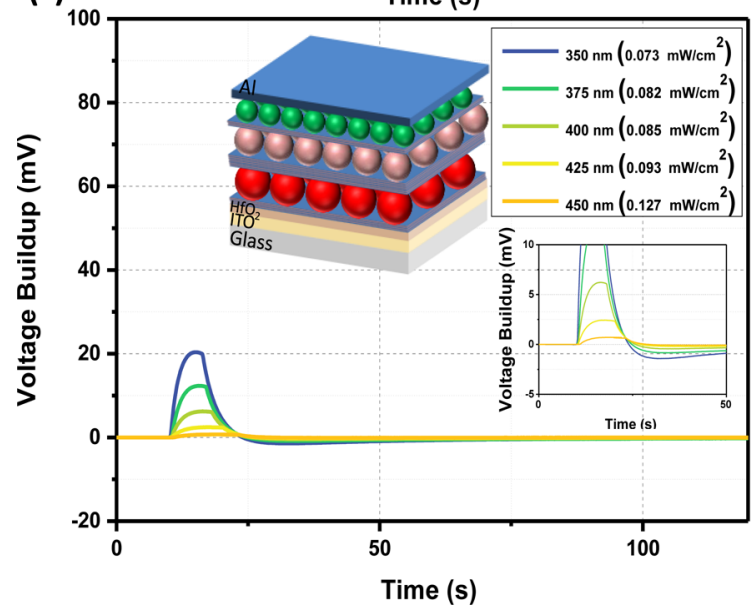

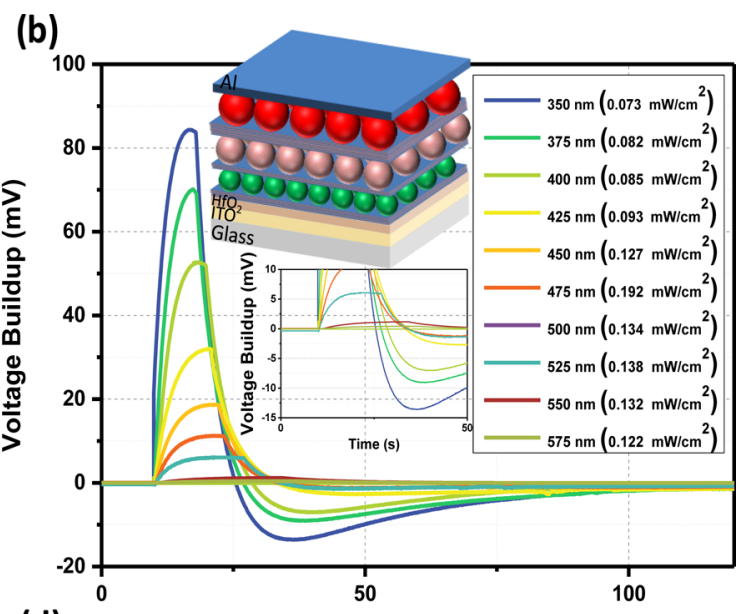

(d)

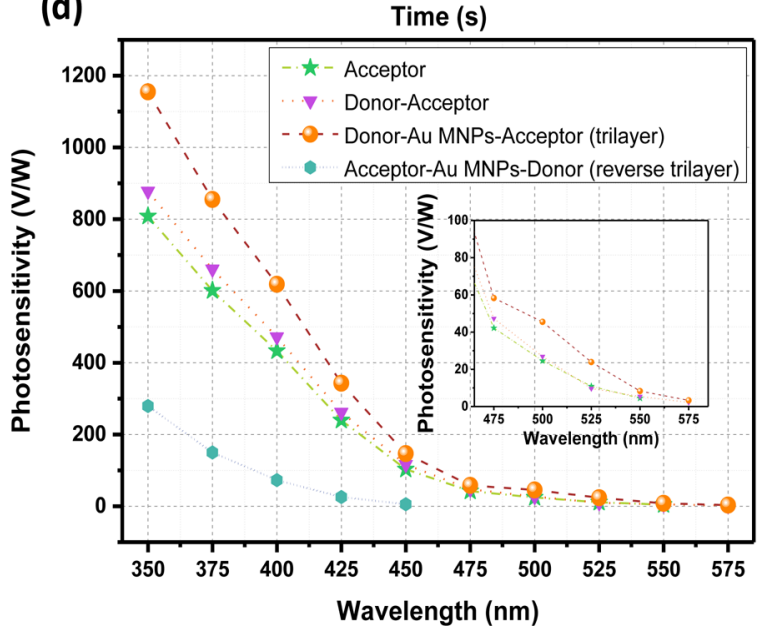

Figure 5. Photovoltage build-up at various excitation wavelengths and optical intensities for (a) donor-acceptor bilayer, (b) trilayer, (c) reverse trilayer, and (d) comparison of the corresponding sensitivities as a function of the excitation wavelength for the acceptor, the donoracceptor bilayer, the trilayer, and its reversed one. Significant enhancement in voltage build-up and photosensitivity in the trilayer structure are attributed to the LSP-enhanced FRET.

function of the time across seven devices with different structures are presented in Figures 4 and 5. In all cases, the voltage build-up enhancement/suppression is calculated from the ratio of peak values. For the device employing a single donor NC layer, we show change in the photovoltage build-up levels at different excitation wavelengths (Figure $4 \mathrm{a}$ ). In order to explore the localized plasmon interaction in the proximity of MNPs, we put 3 MLs of PDDA/PSS pairs between donor NCs and $\mathrm{Au}$ MNPs. With respect to the only donor device, the $\mathrm{Au}$ MNPs-donor device shows a reduction in the voltage build-up (Figure 4b) due to enhancement of nonradiative channels by $\mathrm{Au} \mathrm{MNPs}$ and consequently reduction in sensitivity of the device (see Supporting Information Figure S6). Diminishing voltage build-up can be explained by quenching due to the insufficient interspace distance between NCs and MNPs, which influences the charges trapped inside NCs and migration of the holes to the $\mathrm{Al}$ contact. Hence, the only donor sample has a detectable voltage buildup of $0.89 \mathrm{mV}$ around $550 \mathrm{~nm}$ wavelength; however, in the donor-Au MNPs structure the operational wavelength range is limited to $475 \mathrm{~nm}$ wavelength $(0.86 \mathrm{mV})$ caused by repression of charge accumulation. On the other hand, in comparison to the only acceptor and acceptor- $\mathrm{Au} \mathrm{MNPs}$ devices, the enhancement in the voltage build-up can be clearly seen in Figure 4c,d. This improvement is based on the reduced quenching of excitons via increasing the spacing between NCs and MNPs ( 7 MLs PDDA/PSS pairs). As a result, in the presence of $\mathrm{Au}$ MNPs, there is an enhancement in optical absorption of the NCs film, resulting in increasing the electron and hole pairs generation. Respectively, a larger voltage build-up is observed (up to 2.38-fold enhancement) which consequently enhances the sensitivity of the device (Figure $4 \mathrm{c}, \mathrm{d}$ and Supporting Information Figure S7). Similarly, for the plasmonic device structure, a voltage build-up is observed at longer wavelengths (up to $575 \mathrm{~nm}$ ), which indicates the absorption enhancement in NCs. Moreover, at around $525 \mathrm{~nm}$ excitation wavelength, the acceptor-Au MNPs device shows a significant enhancement in the photosensitivity, which agrees well with the Au MNPs LSP resonance peak.

Next, we examined the device in which FRET is the only active process. The donor and the acceptor NCs in this structure were interspaced by 17 MLs of PDDA/PSS pairs with a total thickness of $t_{\mathrm{D}-\mathrm{A}} \approx 22 \mathrm{~nm}$, which was equivalent to the surface-to-surface donor-acceptor separation in the trilayer structure. The voltage build-up in the donor-acceptor bilayer sample (Figure 5a) was slightly higher than only acceptor reference sample (Figure 4c) because of an inefficient FRET mechanism due to large NCs separation (see Supporting Information Figure S8 for more details). Then, we looked at the trilayer structure, which showed a considerable enhance- 
ment in the voltage build-up, photosensitivity, and extension of operating wavelengths (see Figure $5 b$ ). The spotted enhancement in the trilayer structure's performance can be accredited to the LSP-enhanced FRET. The presence of MNPs results in an increased FRET efficiency between the donor and acceptor NCs, and subsequently more excitons were generated at the NCs layer underneath the $\mathrm{Al}$ electrode. These electron hole pairs dissociated, and the holes accumulated at the electrode (Al contact), resulting in a larger voltage build-up. Likewise, more electrons get trapped in the NCs, as perceived from the larger negative voltage levels of the photovoltage build-up. This consequently enhanced the photovoltage build-up by a factor of up to 1.4 compared to that of an only acceptor device. The trilayer structure revealed a significant enhancement in the voltage build-up and sensitivity around $525 \mathrm{~nm}$ with a 2.91 -fold enhancement, which agreed properly with the LSP resonance peak of $\mathrm{Au}$ MNPs. Additionally, the trilayer NC monolayer structure exhibited considerable extension in its operating wavelength range to $575 \mathrm{~nm}$. With respect to the acceptor monolayer structure with $\mathrm{Au}$ MNPs, the trilayer structure's photovoltage build-up showed an increase up to 1.9 -fold. This observation suggests the strong impact of the LSP-enhanced FRET in the proposed device architecture. Hence, in comparison to the donor-acceptor NCs bilayer sample, we observed an approximately 2-fold enhancement in the voltage buildup of the trilayer device around the Au MNPs LSP, indicating the effect of MNPs on the energy-transfer process (see Supporting Information Figure S9 for more details).

To investigate the direction of the energy transfer, we fabricated a reversed structure whose voltage build-up is presented in Figure 5c. For this structure, the places of the donor NCs and the acceptor NCs were exchanged; however, keeping the rest of the device structure identical to the trilayer structure, $1 \mathrm{ML}$ of donor NCs was interspaced by $3 \mathrm{MLs}$ of PDDA/PSS pair from a single layer of Au MNPs. Similarly, 1 ML of acceptor NCs was interspaced by 7 MLs of PDDA/PSS from $1 \mathrm{ML}$ of $\mathrm{Au}$ MNPs. The inferior performance of the reversed trilayer device was ascribed to the depopulation of photogenerated electron hole pairs from the monolayer of donor NCs because of the energy transfer in the opposite direction. So, fewer number of holes accumulated at the $\mathrm{Al}$ electrode, and accordingly, fewer number of electrons trapped in the NC layer beneath the electrode. As seen in Figure 5c, there is a noticeable difference between the reversed structure and the only donor device (see Supporting Information Figure S10 for more details). More than a 3 -fold suppression in the voltage buildup of the reversed device and its operation wavelength range reduction $(350-450 \mathrm{~nm})$ indicated that a large number of excitons were transferred to the acceptor NCs rather than migrated to the $\mathrm{Al}$ electrode. To this end, lower performance of the acceptor-Au MNPs-donor (reversed trilayer) device compared to the $\mathrm{Au} \mathrm{MNP}$-donor device suggested that the quenching effect and exciton transfer between the NCs were responsible for this performance reduction. Subsequently, these observations strongly coincide with the proposed LSP-enhanced FRET process from the monolayer of a donor toward the acceptor NCs. Figure $5 \mathrm{~d}$ presents the photosensitivity comparison between the acceptor, the donor-acceptor bilayer, the acceptor-Au MNPs-donor (trilayer), and acceptor-Au MNPs-donor (reversed trilayer)based devices as a function of the excitation wavelength. As it can be clearly seen, the performance of devices is in good agreement with the optical absorption spectra of NCs.
Likewise, there is a lower negative voltage value at longer excitation wavelengths due to the less number of photogenerated charges, attributable to the absorption spectra of NCs. Furthermore, at longer wavelengths (above $600 \mathrm{~nm}$ ), NCs show absorption, however, none of the device show any photosensitivity at that range as the number of photogenerated electron-hole pairs are not sufficient.

\section{CONCLUSION}

In this article, $\mathrm{Au}-\mathrm{MNP}$-assisted nonradiative energy transfer resulted in a significant increase in the population of photogenerated excitons, enhancing the sensitivity of the device and extending operating wavelength range. The obtained experimental observations and numerical simulations exhibited that the enhancement in LSP-enhanced FRET device was superior compared to the FRET-based device without plasmon coupling. This performance improvement was attributed to the strong surface plasmon-exciton interactions in the donor-Au MNPs-acceptor layered architecture interspaced by polyelectrolyte spacer layers. Furthermore, by conducting PL and TRF measurements of the trilayer structure, we observed the reduction in PL and average lifetime of the donor NCs, along with the increase in PL and average lifetime of the acceptor NCs, and attributed these behaviors to the LSP-enhanced FRET mechanism. These observations strongly agreed with the device's photovoltage build-up readout, photosensitivity enhancement, and operating wavelength range extension in the case of using LSP-enhanced FRET in the device operation. Such a coupling of the FRET mechanism with plasmonics and its combined utilization in NC-based photovoltage controlled devices hold great promise for nanophotonic device applications.

\section{METHODS}

Chemicals. Cadmium oxide (CdO, 99.99\%), zinc acetate ( $\mathrm{Zn}$ (acetate) $\left.)_{2}, 99.9 \%\right)$, sulfur (S, 99.9\%), and selenium (Se, 99.99\%) were supplied from Sigma-Aldrich in powder form. Oleic acid (OA, 90\%), trioctylphosphine (TOP, 90\%), 1-octadecene (1-ODE, 90\%), dodecanethiol (DDT, 99\%), and chloroform were bought from Sigma-Aldrich. All chemicals were used without further purification, and Milli-Q water was used throughout.

Colloidal Synthesis of Au MNPs. TGA-capped Au MNPs were synthesized by mixing $8 \mathrm{~mL}$ ethanol with $2 \mathrm{~mL}$ of $2 \mathrm{~g} / \mathrm{L} \mathrm{HAuCl}_{4}$. $3 \mathrm{H}_{2} \mathrm{O}$. Then $10 \mu \mathrm{L}$ of TGA was added into the solution. After mixing for $15 \mathrm{~min}, 4 \mathrm{mg}$ of $\mathrm{NaBH}_{4}$ in $1 \mathrm{~mL}$ of Milli-Q water was injected quickly into the vigorously stirring solution. The solution was kept for $5 \mathrm{~min}$ and then cleaned via addition of isopropanol/centrifuging for three times. The resulting nanoparticles formed stable colloidal aqueous solutions and were stable in the solution without any coagulated particles at the bottom of vials for around two months. We observed that they were even stable in low concentration $\mathrm{NaCl}$-water mixtures, which were generally utilized for the LBL coating process where citrate-stabilized Au nanoparticles fail. 50,51

Colloidal Synthesis of CdSe/CdZnS NCs. Green-emitting NCs were synthesized according to a recipe of W. K. Bae et al. ${ }^{39}$ First, 0.4 mmol of cadmium oxide and $4 \mathrm{mmol}$ of zinc acetate were put into a $100 \mathrm{~mL}$ three-neck flask. Then, $17.6 \mathrm{mmol}$ of oleic acid and $20 \mathrm{~mL}$ of 1 -octadecene were added to the flask, and the temperature was raised to $150{ }^{\circ} \mathrm{C}$ under reduced pressure to eliminate the oxygen and moisture. After $30 \mathrm{~min}$, the flask was filled with argon gas, and the temperature was further raised to $310^{\circ} \mathrm{C}$. Meanwhile, a mixture of 0.1 mmol of elemental selenium and $4 \mathrm{mmol}$ of sulfur powder in $3 \mathrm{~mL}$ of TOP was prepared in a $\mathrm{N}_{2}$-filled glovebox with stirring. When the temperature of the flask reached $310^{\circ} \mathrm{C}$, this mixture was injected into the hot solution swiftly. After the NCs reached the desired emission peak wavelength, the growth was stopped by cooling the solution 
down to the room temperature. NCs were cleaned with the addition of acetone and centrifugation. Then, NCs were dissolved in pure hexane for further studies.

For the red-emitting NCs, we followed a slightly modified version of the Z. Soran-Erdem et al. ${ }^{52} 1 \mathrm{mmol}$ of cadmium oxide and $2 \mathrm{mmol}$ of zinc acetate were put into a flask. Then, $2.5 \mathrm{~mL}$ of oleic acid and 25 $\mathrm{mL}$ of 1-octadecene were added. To remove the oxygen and moisture from the flask, it was degassed under vacuum at $100^{\circ} \mathrm{C}$ for $2 \mathrm{~h}$. After degassing, the flask was filled with pure argon gas and heated to 310 ${ }^{\circ} \mathrm{C}$. A solution of $0.2 \mathrm{mmol}$ of elemental selenium in $0.2 \mathrm{~mL}$ of TOP and $0.8 \mathrm{mmol}$ of 1-ocadecene was prepared in a glovebox, and it was injected to the hot solution quickly. After a minute, a mixture of 0.3 $\mathrm{mL}$ of DDT and $0.8 \mathrm{mmol}$ of ODE was injected into the flask dropwise. After $20 \mathrm{~min}$ of growth, the second shell layer was deposited by injecting a solution of $2 \mathrm{mmol}$ of sulfur powder in $1 \mathrm{~mL}$ of TOP into the growth mixture. An additional $10 \mathrm{~min}$ of growth was performed, and the flask was brought to room temperature. Assynthesized NCs were cleaned with the addition of acetone and centrifugation. Then, NCs were dissolved in pure hexane for further studies.

Ligand Exchange of CdSe/CdZnS NCs. To benefit from the LBL techniques, we performed ligand exchange and obtained water-soluble NCs according to the following recipe: As the first step, $15 \mathrm{~mL}$ of pure methanol and $250 \mu \mathrm{L}$ of MPA were mixed. Then, $30 \mathrm{~mL}$ of pure hexane was added, and the mixture was placed in a sonicator. A dispersion of NCs in $2 \mathrm{~mL}$ of hexane was prepared and added to the mixture dropwise. After $30 \mathrm{~min}$ of sonication, it was centrifuged, and the supernatant was discarded. The precipitate was dissolved in a minimum amount of Milli- $\mathrm{Q}$ water. Then, pure acetone was added, and the mixture was centrifuged to clean the excess MPA. The precipitate was dissolved in Milli- $Q$ water, filtered, and used for the LBL assembly.

Time-Resolved Fluorescence Measurements. TRF measurements were recorded using a pulsed laser excitation of $375 \mathrm{~nm}(3.3$ $\mathrm{eV}$ ) with a pulse width $<50$ ps at a repetition rate of $5 \mathrm{MHz}$, focused into a spot size of $37 \mu \mathrm{m}^{2}$ on the sample. To stay in the single exciton regime operation, the excitation fluency was intentionally adjusted low enough at around $5 \times 10^{12}$ photons $/ \mathrm{cm}^{2}$ per pulse. The multiexponential fitting via deconvolution of the instrument response function which led to the best $\chi^{2}$ values was used to fit the TRF decays. To calculate the amplitude-averaged lifetime, following equation was used $\tau_{\text {avg }}=\left(\Sigma\left(A_{\mathrm{i}} \tau_{\mathrm{i}}\right)\right) /\left(\Sigma\left(\mathrm{A}_{\mathrm{i}}\right)\right)$.

\section{ASSOCIATED CONTENT}

\section{S Supporting Information}

The Supporting Information is available free of charge on the ACS Publications website at DOI: 10.1021/acsnano.6b08392.

Further descriptions on the device fabrication, characterization, and LBL assembly method (S1); TEM images of NCs and MNPs (S2); NCs TRF decay curves (S3); Additional details on the theoretical model (S4); Device operational mechanism (S5); Photovoltage build-up comparison between devices of: donor and Au MNPdonor (S6); acceptor and Au MNP-acceptor (S7); acceptor and donor-acceptor (S8); acceptor and donor-Au MNP-acceptor (S9); donor and acceptor$\mathrm{Au} \mathrm{MNP}-$ donor (S10) (PDF)

\section{AUTHOR INFORMATION}

\section{Corresponding Author}

*E-mail: hvdemir@ntu.edu.sg; volkan@stanfordalumni.org. ORCID

Hilmi Volkan Demir: 0000-0003-1793-112X

\section{Present Addresses}

${ }^{\S}$ Department of Engineering, University of Cambridge, Cambridge CB30FA, United Kingdom
"ICFO-Institut de Ciencies Fotoniques, The Barcelona Institute of Science and Technology, Castelldefels, Barcelona 08860, Spain

\section{Author Contributions}

${ }^{\#}$ These authors contributed equally to this work.

\section{Notes}

The authors declare no competing financial interest.

\section{ACKNOWLEDGMENTS}

We gratefully acknowledge the financial support in part by TUBITAK under the project no. EEEAG 114E449 and partly from NRFI2016-08. HVD acknowledges additional support from TUBA.

\section{REFERENCES}

(1) Förster, T. Intermolecular Energy Migration and Fluorescence. Ann. Phys. (Berlin, Ger.) 1948, 437, 55-75.

(2) Yeltik, A.; Guzelturk, B.; Hernandez-Martinez, P. L.; Akhavan, S.; Demir, H. volkan. Excitonic Enhancement of Nonradiative Energy Transfer to Bulk Silicon with the Hybridization of Cascaded Quantum Dots. Appl. Phys. Lett. 2013, 103, 261103.

(3) Chanyawadee, S.; Lagoudakis, P. G.; Harley, R. T.; Charlton, M. D. B.; Talapin, D. V.; Huang, H. W.; Lin, C. H. Increased ColorConversion Efficiency in Hybrid Light-Emitting Diodes utilizing NonRadiative Energy Transfer. Adv. Mater. 2009, 22, 602-606.

(4) Achermann, M.; Petruska, M. A.; Kos, S.; Smith, D. L.; Koleske, D. D.; Klimov, V. I. Energy-Transfer Pumping of Semiconductor Nanocrystals Using an Epitaxial Quantum Well. Nature 2004, 429, 642-646.

(5) Cicek, N.; Nizamoglu, S.; Ozel, T.; Mutlugun, E.; Karatay, D. U.; Lesnyak, V.; Otto, T.; Gaponik, N.; Eychmüiller, A.; Demir, H. V. Structural Tuning of Color Chromaticity Through Nonradiative Energy Transfer by Interspacing CdTe Nanocrystal Monolayers. Appl. Phys. Lett. 2009, 94, 061105.

(6) Nizamoglu, S.; Sari, E.; Baek, J.-H.; Lee, I.-H.; Volkan Demir, H. White light Generation by Resonant Nonradiative Energy Transfer from Epitaxial InGaN/GaN Quantum Wells to Colloidal CdSe/ZnS Core/Shell Quantum Dots. New J. Phys. 2008, 10, 123001.

(7) Nizamoglu, S.; Demir, H. V. Resonant Nonradiative Energy Transfer in CdSe/ZnS Core/Shell Nanocrystal Solids Enhances Hybrid White Light Emitting Diodes. Opt. Express 2008, 16, 13961-13968.

(8) Miyawaki, a; Llopis, J.; Heim, R; McCaffery, J. M.; Adams, J. a; Ikura, M.; Tsien, R. Y. Fluorescent Indicators for Ca2+Based on Green Fluorescent Proteins and Calmodulin. Nature 1997, 388, 882-887.

(9) Medintz, I. L.; Clapp, A. R.; Mattoussi, H.; Goldman, E. R.; Fisher, B.; Mauro, J. M. Self-Assembled Nanoscale Biosensors Based on Quantum Dot FRET Donors. Nat. Mater. 2003, 2, 630-638.

(10) Gaponenko, S. V. Introduction to Nanophotonics; Cambridge University Press: Cambridge, U.K., 2010.

(11) Akhavan, S.; Cihan, A. F.; Bozok, B.; Demir, H. V. Nanocrystal Skins with Exciton Funneling for Photosensing. Small 2014, 10, $2470-2475$

(12) Lunz, M.; Bradley, A. L.; Chen, W. Y.; Gun'ko, Y. K. TwoDimensional Förster Resonant Energy Transfer in a Mixed Quantum Dot Monolayer: Experiment and Theory. J. Phys. Chem. C 2009, 113, 3084-3088.

(13) Scholes, G.; Andrews, D. Resonance Energy Transfer and Quantum Dots. Phys. Rev. B: Condens. Matter Mater. Phys. 2005, 72, 125331.

(14) Lunz, M.; Bradley, a. L.; Chen, W.-Y.; Gerard, V. a.; Byrne, S. J.; Gun'ko, Y. K.; Lesnyak, V.; Gaponik, N. Influence of Quantum Dot Concentration on Förster Resonant Energy Transfer in Monodispersed Nanocrystal Quantum Dot Monolayers. Phys. Rev. B: Condens. Matter Mater. Phys. 2010, 81, 205316.

(15) Kagan, C.; Murray, C.; Bawendi, M. Long-Range Resonance Transfer of Electronic Excitations in Close-Packed CdSe Quantum- 
Dot Solids. Phys. Rev. B: Condens. Matter Mater. Phys. 1996, 54, 86338643.

(16) Shimizu, K. T.; Woo, W. K.; Fisher, B. R.; Eisler, H. J.; Bawendi, M. G. Surface-Enhanced Emission from Single Semiconductor Nanocrystals. Phys. Rev. Lett. 2002, 89, 117401.

(17) Ozel, T.; Hernandez-Martinez, P. L.; Mutlugun, E.; Akin, O.; Nizamoglu, S.; Ozel, I. O.; Zhang, Q.; Xiong, Q.; Demir, H. V. Observation of Selective Plasmon-Exciton Coupling in Nonradiative Energy Transfer: Donor-Selective versus Acceptor-Selective Plexcitons. Nano Lett. 2013, 13, 3065-3072.

(18) Zhang, X.; Marocico, C. A.; Lunz, M.; Gerard, V. A.; Gun'Ko, Y. K.; Lesnyak, V.; Gaponik, N.; Susha, A. S.; Rogach, A. L.; Bradley, A. L. Experimental and Theoretical Investigation of the Distance Dependence of Localized Surface Plasmon Coupled Förster Resonance Energy Transfer. ACS Nano 2014, 8, 1273-1283.

(19) Ming, T.; Chen, H.; Jiang, R.; Li, Q.; Wang, J. PlasmonControlled Fluorescence: Beyond the Intensity Enhancement. J. Phys. Chem. Lett. 2012, 3, 191-202.

(20) Andrew, P.; Barnes, W. L. Energy Transfer Across a Metal Film Mediated by Surface Plasmon Polaritons. Science 2004, 306, 10021005 .

(21) Su, X.-R.; Zhang, W.; Zhou, L.; Peng, X.-N.; Wang, Q.-Q. Plasmon-Enhanced Förster Energy Transfer Between Semiconductor Quantum Dots: Multipole Effects. Opt. Express 2010, 18, 6516-6521.

(22) Su, X.-R.; Zhang, W.; Zhou, L.; Peng, X.-N.; Pang, D.-W.; Liu, S.-D.; Zhou, Z.-K.; Wang, Q.-Q. Multipole-Plasmon-Enhanced Förster Energy Transfer Between Semiconductor Quantum Dots via DualResonance Nanoantenna Effects. Appl. Phys. Lett. 2010, 96, 043106.

(23) Fofang, N. T.; Park, T. H.; Neumann, O.; Mirin, N. A.; Nordlander, P.; Halas, N. J. Plexcitonic Nanoparticles: PlasmonExciton Coupling in Nanoshell-J-Aggregate Complexes. Nano Lett. 2008, 8, 3481-3487.

(24) Manjavacas, A.; Garcia de Abajo, F. J.; Nordlander, P. Quantum Plexcitonics: Strongly Interacting Plasmons and Excitons. Nano Lett. 2011, 11, 2318-2323.

(25) West, R. G.; Sadeghi, S. M. Enhancement of Energy Transfer between Quantum Dots: The Impact of Metallic Nanoparticle Sizes. J. Phys. Chem. C 2012, 116, 20496-20503.

(26) Kim, K. S.; Kim, J. H.; Kim, H.; Laquai, F.; Arifin, E.; Lee, J. K.; Yoo, S. I.; Sohn, B. H. Switching Off FRET in the Hybrid Assemblies of Diblock Copolymer Micelles, Quantum Dots, and Dyes by Plasmonic Nanoparticles. ACS Nano 2012, 6, 5051-5059.

(27) Wang, C. H.; Chen, C. W.; Chen, Y. T.; Wei, C. M.; Chen, Y. F.; Lai, C. W.; Ho, M. L.; Chou, P. T.; Hofmann, M. Surface Plasmon Enhanced Energy Transfer Between Type I CdSe/ZnS and Type II CdSe/ZnTe Quantum Dots. Appl. Phys. Lett. 2010, 96, 071906.

(28) Lessard-Viger, M.; Rioux, M.; Rainville, L.; Boudreau, D. FRET Enhancement in Multilayer Core-Shell Nanoparticles. Nano Lett. 2009, 9, 3066-3071.

(29) Zhao, L.; Ming, T.; Shao, L.; Chen, H.; Wang, J. PlasmonControlled Förster Resonance Energy Transfer. J. Phys. Chem. C 2012, 116, 8287-8296.

(30) Lunz, M.; Gerard, V. A.; Gun'ko, Y. K.; Lesnyak, V.; Gaponik, N.; Susha, A. S.; Rogach, A. L.; Bradley, A. L. Surface Plasmon Enhanced Energy Transfer between Donor and Acceptor CdTe Nanocrystal Quantum Dot Monolayers. Nano Lett. 2011, 11, 33413345 .

(31) Komarala, V. K.; Bradley, A. L.; Rakovich, Y. P.; Byrne, S. J.; Gun'ko, Y. K.; Rogach, A. L. Surface Plasmon Enhanced Förster Resonance Energy Transfer between the CdTe Quantum Dots. Appl. Phys. Lett. 2008, 93, 123102.

(32) Zhang, J.; Fu, Y.; Lakowicz, J. R. Enhanced Förster Resonance Energy Transfer (FRET) on a Single Metal Particle. J. Phys. Chem. C 2007, 111, 50-56.

(33) Viger, M. L.; Brouard, D.; Boudreau, D. Plasmon-Enhanced Resonance Energy Transfer from a Conjugated Polymer to Fluorescent Multilayer Core-Shell Nanoparticles: A Photophysical Study. J. Phys. Chem. C 2011, 115 (7), 2974-2981.
(34) Yang, K. Y.; Choi, K. C.; Ahn, C. W. Surface Plasmon-Enhanced Spontaneous Emission Rate in an Organic Light-Emitting Device Structure: Cathode Structure for Plasmonic Application. Appl. Phys. Lett. 2009, 94, 173301-173303.

(35) Becker, K.; Lupton, J. M.; Müller, J.; Rogach, A. L.; Talapin, D. V.; Weller, H.; Feldmann, J. Electrical Control of Förster Energy Transfer. Nat. Mater. 2006, 5, 777-781.

(36) Lunz, M.; Zhang, X.; Gerard, V. A.; Gun'Ko, Y. K.; Lesnyak, V.; Gaponik, N.; Susha, A. S.; Rogach, A. L.; Bradley, A. L. Effect of Metal Nanoparticle Concentration on Localized Surface Plasmon Mediated Förster Resonant Energy Transfer. J. Phys. Chem. C 2012, 116, 2652926534.

(37) Akhavan, S.; Uran, C.; Bozok, B.; Gungor, K.; Kelestemur, Y.; Lesnyak, V.; Gaponik, N.; Eychmuller, A.; Demir, H. V. Flexible and Fragmentable Tandem Photosensitive Nanocrystal Skins. Nanoscale 2016, 8, 4495-4503.

(38) Akhavan, S.; Yeltik, A.; Demir, H. V. Photosensitivity Enhancement with $\mathrm{TiO} 2$ in Semitransparent Light-Sensitive Skins of Nanocrystal Monolayers. ACS Appl. Mater. Interfaces 2014, 6, 90239028.

(39) Bae, W. K.; Char, K.; Hur, H.; Lee, S. Single-Step Synthesis of Quantum Dots with Chemical Composition Gradients. Chem. Mater. 2008, 20, 531-539.

(40) Martin, M. N.; Basham, J. I.; Chando, P.; Eah, S. K. Charged Gold Nanoparticles in Non-Polar Solvents: 10-min Synthesis and 2D Self-Assembly. Langmuir 2010, 26, 7410-7417.

(41) Soganci, I. M.; Nizamoglu, S.; Mutlugun, E.; Akin, O.; Demir, H. V. Localized Plasmon-Engineered Spontaneous Emission of CdSe/ $\mathrm{ZnS}$ Nanocrystals Closely-Packed in the Proximity of Ag Nanoisland Films for Controlling Emission Linewidth, Peak, and Intensity. Opt. Express 2007, 15, 14289-14298.

(42) Song, J.-H.; Atay, T.; Shi, S.; Urabe, H.; Nurmikko, A. V. Large Enhancement of Fluorescence Efficiency from CdSe/ZnS Quantum Dots Induced by Resonant Coupling to Spatially Controlled Surface Plasmons. Nano Lett. 2005, 5, 1557-1561.

(43) Lakowicz, J. R. Principles of Fluorescence Spectroscopy; Springer: New York, 2006.

(44) Hernandez-Martinez, P. L.; Govorov, A. O.; Demir, H. V. Förster-Type Nonradiative Energy Transfer for Assemblies of Arrayed Nanostructures: Confinement Dimension vs Stacking Dimension. J. Phys. Chem. C 2014, 118, 4951-4958.

(45) Govorov, A. O.; Bryant, G. W.; Zhang, W.; Skeini, T.; Lee, J.; Kotov, N. A.; Slocik, J. M.; Naik, R. R. Exciton-Plasmon Interaction and Hybrid Excitons in Semiconductor-Metal Nanoparticle Assemblies. Nano Lett. 2006, 6, 984-994.

(46) Anikeeva, P.; Madigan, C.; Halpert, J.; Bawendi, M.; Bulović, V. Electronic and Excitonic Processes in Light-Emitting Devices Based on Organic Materials and Colloidal Quantum Dots. Phys. Rev. B: Condens. Matter Mater. Phys. 2008, 78, 1-8.

(47) Akhavan, S.; Guzelturk, B.; Sharma, V. K.; Demir, H. V. Largearea Semi-Transparent Light-Sensitive Nanocrystal Skins. Opt. Express 2012, 20, 25255-2566.

(48) Akhavan, S.; Gungor, K.; Mutlugun, E.; Demir, H. V. Plasmonic Light-Sensitive Skins of Nanocrystal Monolayers. Nanotechnology 2013, 24, 155201.

(49) Akhavan, S.; Cihan, A. F.; Yeltik, A.; Bozok, B.; Lesnyak, V.; Gaponik, N.; Eychmüller, A.; Demir, H. V. Multiexciton Generation Assisted Highly Photosensitive $\mathrm{CdHgTe}$ Nanocrystal Skins. Nano Energy 2016, 26, 324-331.

(50) Shavel, A.; Gaponik, N.; Eychmüller, A. The Assembling of Semiconductor Nanocrystals. Eur. J. Inorg. Chem. 2005, 2005, 36133623.

(51) Gaponik, N. Assemblies of Thiol-Capped Nanocrystals as Building Blocks for Use in Nanotechnology. J. Mater. Chem. 2010, 20, 5174-5181.

(52) Soran-Erdem, Z.; Erdem, T.; Hernandez-Martinez, P. L.; Akgul, M. Z.; Gaponik, N.; Demir, H. V. Macrocrystals of Colloidal Quantum Dots in Anthracene: Exciton Transfer and Polarized Emission. J. Phys. Chem. Lett. 2015, 6, 1767-1772. 